\title{
On Rich Lines in Grids
}

\author{
Evan Borenstein • Ernie Croot
}

Received: 1 October 2008 / Revised: 26 January 2010 / Accepted: 27 January 2010 /

Published online: 12 February 2010

(C) Springer Science+Business Media, LLC 2010

\begin{abstract}
In this paper we show that if one has a grid $A \times B$, where $A$ and $B$ are sets of $n$ real numbers, then there can be only very few "rich" lines in certain quite small families. Indeed, we show that if the family has lines taking on $n^{\varepsilon}$ distinct slopes, and where each line is parallel to $n^{\varepsilon}$ others (so, at least $n^{2 \varepsilon}$ lines in total), then at least one of these lines must fail to be "rich". This result immediately implies nontrivial sumproduct inequalities; though, our proof makes use of the Szemeredi-Trotter inequality, which Elekes used in his argument for lower bounds on $|C+C|+|C . C|$.
\end{abstract}

Keywords Sum-product inequalities · Point-line configurations $\cdot$ Szemerédi-Trotter inequality

\section{Introduction}

In [2], Erdős and Szemerédi proved the following result, which has led to a remarkable number of profound developments in the field of additive combinatorics:

Theorem 1 There is some absolute constant $\varepsilon>0$ such that if $A$ is a set of real numbers, $|A| \geq 2$, then either the sum set $A+A$ or the product set $A$.A, has size at least $|A|^{1+\varepsilon}$.

In [3], Elekes gave a brilliantly elegant proof of this theorem using the SzemerédiTrotter incidence theorem [7], and was able to show that

$$
|A+A| \cdot|A . A| \gg|A|^{5 / 2},
$$

E. Borenstein summer funding supported by an NSF VIGRE grant.

E. Croot supported in part by an NSF grant.

E. Borenstein · E. Croot $(\bowtie)$

School of Mathematics, Georgia Institute of Technology, 103 Skiles, Atlanta, GA 30332, USA

e-mail: ecroot@math.gatech.edu 
from which it follows that

$$
\max (|A+A|,|A \cdot A|) \gg|A|^{5 / 4} .
$$

The key fact that Elekes needed for his proof, and which is a weak corollary of the Szemerédi-Trotter incidence theorem, at least as far as just getting a non-trivial bound of the sort

$$
|A+A| \cdot|A \cdot A| \gg|A|^{2+\varepsilon},
$$

is the following basic claim.

Claim 1 There are absolute constants $\varepsilon>0$ and $\delta>0$ such that if $A$ and $B$ are sets of $n$ real numbers, and $n$ is sufficiently large (in terms of $\varepsilon$ and $\delta$ ), then any set of at least $n^{2-\varepsilon}$ distinct lines contains a member that hits the grid in fewer than $n^{1-\delta}$ points. In other words, one cannot have a collection of $n^{2-\varepsilon}$ lines whereby all are " $n^{1-\delta}$-rich" in the grid $A \times B$.

Actually, Elekes's proof only needs the following even weaker claim.

Claim 2 There exist absolute constants $\varepsilon>0$ and $\delta>0$ so that the following holds for all integers $n$ sufficiently large: Suppose that $A$ and $B$ are sets of real numbers of size $n$, and that one has a family of lines such that

- there are at least $n^{1-\varepsilon}$ distinct slopes among them; and,

- every line is parallel to at least $n^{1-\varepsilon}$ others.

Then, at least one of the lines must hit the grid $A \times B$ in fewer than $n^{1-\delta}$ points. In other words, not all the lines can be $n^{1-\delta}$-rich in the grid.

In the present paper, we prove the following theorem, which shows that it is possible to considerably strengthen this second claim; furthermore, our theorem is not the sort that is quickly deducible from the Szemerédi-Trotter incidence theorem:

Theorem 2 For every $\varepsilon>0$, there exists $\delta>0$ so that the following holds for all $n$ sufficiently large: Suppose that $A$ and $B$ are sets of real numbers of size $n$, and that one has a family of lines such that

- there are at least $n^{\varepsilon}$ distinct slopes among them; and,

- every line is parallel to at least $n^{\varepsilon}$ others.

Then, at least one of the lines must hit the grid $A \times B$ in fewer than $n^{1-\delta}$ points.

Our theorem is related to a conjecture of Solymosi (see [4, Conjecture 3.10] for details), which we modify and extend to make it better fit the context of the above results.

Solymosi's Conjecture For every $\varepsilon>0$, there exists $\delta>0$ such that the following holds for all integers $n$ sufficiently large: Suppose $A$ and $B$ are sets of real numbers of size $n$, and suppose that one has a collection of $n^{\varepsilon}$ lines in general position (that 
is, no pair is parallel, and no three meet at a point). Then, not all of the lines can be $n^{1-\delta}$-rich in the grid $A \times B$.

This conjecture of Solymosi easily implies our main theorem (Theorem 2) above, for if one has a family of lines as described by our theorem, then it is a simple matter to select one line from each of $\gg n^{\varepsilon / 3}$ groups of parallel lines in such a way that one produces a collection in general position (first, select a single line of slope $\lambda_{1}$; then, select a line of slope $\lambda_{2} \neq \lambda_{1}$; then, select a line of slope $\lambda_{3} \notin\left\{\lambda_{1}, \lambda_{2}\right\}$ such that the three lines do not have a common intersection point; then, select a line of slope $\left.\lambda_{4} \notin\left\{\lambda_{1}, \lambda_{2}, \lambda_{3}\right\} \ldots\right)$.

\subsection{Remarks}

Our proof makes use of several standard methods in additive combinatorics, though is quite intricate and technical. In particular, some of our approaches are similar to those appearing in the well-known paper of Bourgain, Katz and Tao [1], as was pointed out to us by P.M. Wood. Even so, we do not assume any results more sophisticated than the Szemerédi-Trotter theorem. It was pointed out to us recently by T. Tao that perhaps we could make use of a particular sum-product ideas of Bourgain to give a simpler proof; however, we decided to present here our original approach.

It is possible that perhaps some of the ideas of H. Helfgott $[5,6]$ might allow us to give a shorter proof, as part of our argument can be phrased in terms of growth and generation in subgroups of $G L_{2}(\mathbb{R})$; however, there are a number of differences between Helfgott's work and ours: First, Helfgott works with special linear groups, not general linear groups of matrices that come up in Sect. 2.1 below. Still, it might be possible to modify Helfgott's proof to handle this. Another way that our results differ is that Helfgott works in $\mathbb{Z}_{p}$, whereas we work over $\mathbb{R}$. To get around this problem one can perhaps use the transference theorems of [8], to move the problem from $\mathbb{R}$ to $\mathbb{Z}_{p}$. Another way that our arguments differ from Helfgott's is that our matrix products are of a very special form, and are not the full set of possible products that one generates starting with a seed set; so, to apply Helfgott's ideas, one would need to add some of our "energy lemmas" to his arguments. Finally, we do not make use of commutativity of certain matrix products as Helfgott does (Helfgott reduces to the commutative case) either explicitly or even implicitly; in fact, it is not obvious how one could reduce to this case using our methods (in our first attempt to prove our main theorem we tried to reduce to the commutative case, but were unable to do so for various reasons).

\section{Proof of the Main Theorem}

The first step in our proof is to reduce from the case of working with grids $A \times B$ to grids $A \times A$. This is easily handled by simply letting $C=A \cup B$, and then noting that the hypotheses of our theorem imply that we have a family of rich lines passing through the grid $C \times C$. Upon rescaling $n$ to $|A \cup B| \leq 2 n$, we see that we could have just assumed that our grid was $A \times A$ (or $C \times C$ ) all along. 


\subsection{Producing New Rich Lines from Old Ones}

In our proof, we will be combining together lots of pairs of rich lines, possibly of different slope: Given a line $\ell$ hitting $A \times A$ in some points, we let

$$
\begin{aligned}
& X(\ell)=\text { projection of } \ell \cap(A \times A) \text { onto the } x \text {-axis; } \\
& Y(\ell)=\text { projection of } \ell \cap(A \times A) \text { onto the } y \text {-axis. }
\end{aligned}
$$

If two lines

$$
\ell: y=\lambda x+\mu \quad \text { and } \quad \ell^{\prime}: y=\lambda^{\prime} x+\mu^{\prime}
$$

have the property that

$$
\left|Y(\ell) \cap Y\left(\ell^{\prime}\right)\right|=\text { "large", }
$$

then there will be lots of triples

$$
(x, z, y) \in A \times A \times A
$$

satisfying

$$
\lambda x+\mu=y=\lambda^{\prime} z+\mu^{\prime} .
$$

So, the new line

$$
z=\left(\lambda / \lambda^{\prime}\right) x+\left(\mu-\mu^{\prime}\right) / \lambda^{\prime}
$$

also hits the grid $A \times A$ in many points.

A convenient way of keeping track of the new rich lines that we can produce from old ones is to use matrix notation: We form the association

$$
y=\lambda x+\mu \leftrightarrow\left[\begin{array}{cc}
\lambda & \mu \\
0 & 1
\end{array}\right] .
$$

Then, when we combine together lines as above, the new line we get will be the one associated to a certain product of matrices; specifically,

$$
\begin{aligned}
y & =\left(\lambda / \lambda^{\prime}\right) x+\left(\mu-\mu^{\prime}\right) / \lambda^{\prime} \\
& \leftrightarrow\left[\begin{array}{cc}
\lambda / \lambda^{\prime} & \left(\mu-\mu^{\prime}\right) / \lambda^{\prime} \\
0 & 1
\end{array}\right]=\left[\begin{array}{cc}
\lambda^{\prime} & \mu^{\prime} \\
0 & 1
\end{array}\right]^{-1}\left[\begin{array}{cc}
\lambda & \mu \\
0 & 1
\end{array}\right] .
\end{aligned}
$$

A basic fact, which is an easy consequence of the Cauchy-Schwarz inequality, is the following lemma:

\section{Lemma 1 Given lines}

$$
\ell_{1}, \ldots, \ell_{K}
$$

each hitting a grid

$$
A \times A
$$


in at least

$$
n^{1-\delta_{0}} \text { points, }
$$

we have that at least

$$
K^{2} n^{-2 \delta_{0}} / 2
$$

of the pairs $\left(\ell_{i}, \ell_{j}\right)$ have the property that

$$
\left|Y\left(\ell_{i}\right) \cap Y\left(\ell_{j}\right)\right| \geq n^{1-2 \delta_{0}} / 2 .
$$

If the lines $\ell_{1}, \ldots, \ell_{K}$ have slopes $\lambda_{1}, \ldots, \lambda_{K}$, respectively, then what this lemma is telling us is that we get lots of lines of slope $\lambda_{i} / \lambda_{j}$, for lots of pairs $(i, j)$, such that each is at least $n^{1-2 \delta_{0}} / 2$ rich in the grid $A \times A$.

\subsection{Passing to a Set of Rich Lines with Usable Properties}

Given $\varepsilon>0$, we let $\delta^{\prime}>0$ denote some parameter that we will choose later. Then, given $\varepsilon, \delta^{\prime}>0$ we let $\delta>0$ be some parameter chosen later. We will show that if $\delta>0$ is small enough, and if (as stated in the hypotheses of our theorem) we had a set of lines involving $n^{\varepsilon}$ slopes, each parallel to at least $n^{\varepsilon}$ others, each $n^{1-\delta}$-rich in the grid, then in fact there would have to exist at least $n^{4}$ lines, each hitting $A \times A$ in at least two points. This clearly cannot happen because there are fewer lines hitting the grid in two points than there are ordered pairs of points of the grid-there are $n^{2}$ points of the grid, and therefore $n^{4}$ ordered pairs. This will prove our theorem.

So, we assume that $\varepsilon>0$ is given, and then we will select $\delta^{\prime}>0$ as small as needed, and then choose $\delta>0$ even smaller later.

We begin by letting $L_{1}(\lambda)$ denote the set of our lines having slope $\lambda$. We note that

$$
\left|L_{1}(\lambda)\right| \geq n^{\varepsilon}
$$

To make certain later estimates easier, we will trim our list of lines so that for each slope $\lambda$ we have

$$
\left|L_{1}(\lambda)\right|=\left\lceil n^{\varepsilon}\right\rceil .
$$

Denote our initial set of slopes by $\Lambda_{1}$.

Using Lemma 1, we can easily deduce that there are at least

$$
\left|\Lambda_{1}\right|^{2} n^{-O(\delta)}
$$

ordered pairs

$$
\left(\lambda, \lambda^{\prime}\right) \in \Lambda_{1} \times \Lambda_{1}
$$

for which there are at least

$$
\left|L_{1}(\lambda)\right| \cdot\left|L_{1}\left(\lambda^{\prime}\right)\right| n^{-O(\delta)} \sim n^{2 \varepsilon-O(\delta)}
$$

pairs of lines

$$
\left(\ell, \ell^{\prime}\right) \in L_{1}(\lambda) \times L_{1}\left(\lambda^{\prime}\right)
$$


satisfying

$$
\left|Y(\ell) \cap Y\left(\ell^{\prime}\right)\right| \geq n^{1-O(\delta)} .
$$

Note that each of these intersections gives rise to a line having slope $\lambda / \lambda^{\prime}$ that hits $A \times A$ in $n^{1-O(\delta)}$ points.

When such a pair $\left(\lambda, \lambda^{\prime}\right)$ has the above property we will say that it is "good for step 1". Note that our definition of "good" is dependent upon the implied constants in the big-ohs-for our purposes, the implied constants in the "good for step $i$ " can all be taken to be $1000^{i}$.

If a pair $\left(\lambda, \lambda^{\prime}\right)$ is good for step 1 , and if in addition we have that the number of distinct lines of slope $\lambda / \lambda^{\prime}$ produced by combining pairs $\left(\ell, \ell^{\prime}\right)$ satisfying (2) and (3) is at least

$$
n^{\varepsilon\left(1+\delta^{\prime}\right)}
$$

we will say that $\left(\lambda, \lambda^{\prime}\right)$ is "very good for step 1 ".

Let us suppose that all but at least a fraction $n^{-O(\delta)}$ of the "good" pairs $\left(\lambda, \lambda^{\prime}\right)$ are, in fact, "very good". Let $\Lambda_{2}$ denote these "very good" pairs, and note that we are saying

$$
\left.\left|\Lambda_{2}\right| \geq \mid \text { \{good pairs }\right\}\left.\left|n^{-O(\delta)} \geq\right| \Lambda_{1}\right|^{2} n^{-O(\delta)} .
$$

For $\theta \in \Lambda_{2}$, say $\theta=\left(\lambda, \lambda^{\prime}\right)$, we let $L_{2}(\theta)$ denote those lines produced by combining together pairs of lines, one from $L_{1}(\lambda)$ and the other from $L_{1}\left(\lambda^{\prime}\right)$. Note that for all $\theta \in \Lambda_{2}$ we have, by (4), that

$$
\left|L_{2}(\theta)\right| \geq n^{\varepsilon\left(1+\delta^{\prime}\right)} .
$$

And, as with the set of lines $L_{1}(\lambda)$, we trim our set of lines (in an arbitrary manner) so that for every such $\theta$ we have that

$$
\left|L_{2}(\theta)\right|=\left\lceil n^{\varepsilon\left(1+\delta^{\prime}\right)}\right\rceil .
$$

It is easily deduced from Lemma 1 that there are at least

$$
\left|\Lambda_{2}\right|^{2} n^{-O(\delta)}
$$

ordered pairs

$$
\left(\theta, \theta^{\prime}\right) \in \Lambda_{2} \times \Lambda_{2}
$$

for which there are at least

$$
\left|L_{2}(\theta)\right| \cdot\left|L_{2}\left(\theta^{\prime}\right)\right| n^{-O(\delta)} \sim n^{2 \varepsilon\left(1+\delta^{\prime}\right)-O(\delta)}
$$

pairs of lines

$$
\left(\ell, \ell^{\prime}\right) \in L_{2}(\lambda) \times L_{2}\left(\lambda^{\prime}\right)
$$

satisfying

$$
\left|Y(\ell) \cap Y\left(\ell^{\prime}\right)\right| \geq n^{1-O(\delta)} .
$$


When such a pair $\left(\theta, \theta^{\prime}\right)$ has the above property we will say that it is "good for step 2", and we say that it is "very good for step 2" if the set of rich lines that it produces has size at least

$$
n^{\varepsilon\left(1+\delta^{\prime}\right)^{2}}
$$

We will repeat the above process we have started as above, by defining $\Lambda_{3}$ to be the set of all "very good for step 2" pairs $\beta=\left(\theta, \theta^{\prime}\right) \in \Lambda_{2} \times \Lambda_{2}$, and we will let $L_{3}(\beta)$ be those lines produced by combining together ones from $L_{2}(\theta)$ with $L\left(\theta^{\prime}\right)$, and then trimming the list so that

$$
\left|L_{3}(\beta)\right|=\left\lceil n^{\varepsilon\left(1+\delta^{\prime}\right)^{2}}\right\rceil .
$$

It is clear that we can continue the above process, producing sets

$$
\Lambda_{4}, \quad \Lambda_{5}, \quad \ldots, \quad \text { where } \Lambda_{i} \subseteq \Lambda_{i-1} \times \Lambda_{i-1},
$$

and sets

$$
L_{3}\left(\alpha_{3}\right), \quad L_{4}\left(\alpha_{4}\right), \quad \ldots, \quad \text { where } \alpha_{i} \in \Lambda_{i} .
$$

However, the process cannot go on for too long since we always have the upper bound

$$
\left|L_{t}(\alpha)\right| \leq n^{4}
$$

since the lines of $L_{t}(\alpha)$ will hit the grid in at least two points. In fact,

$$
t \ll T:=\left(1 / \delta^{\prime}\right) \log (4 / \varepsilon) .
$$

Well, the above sequence of $\Lambda_{j}$ 's and $L_{j}\left(\alpha_{j}\right)$ 's is not quite what we want because for later arguments we will need that the sequence terminates with $t>k$, for some $k=k(\varepsilon)$ depending only on $\varepsilon>0$. The way we get around this is as follows: Going back to how our sequences of $\Lambda_{j}$ 's and $L_{j}\left(\alpha_{j}\right)$ 's are defined, if we are willing to allow the $\Lambda_{j}, j=1,2, \ldots, k$, to merely contain "good for step $j$ " pairs, instead of "very good for step $j$ " pairs, then the problem of stopping at time $t \leq k$ is avoided. There is the issue of how to trim the sets $L_{2}\left(\alpha_{2}\right), \ldots, L_{k}\left(\alpha_{k}\right)$ in the right way. To solve this problem, we merely trim them so that they each contain $n^{\varepsilon-O(\delta)}$ lines, which is easily guaranteed. Furthermore, by choosing $\delta^{\prime}>0$ small enough, we can still have that for $j>k$ and $\theta \in \Lambda_{j}$,

$$
\left|L_{j}(\theta)\right|=\left\lceil n^{\varepsilon\left(1+\delta^{\prime}\right)^{j}}\right\rceil,
$$

the reason being that for small $\delta^{\prime}>0$, the $\left(1+\delta^{\prime}\right)^{k}$ can be made as close to 1 as needed.

Before unraveling what this all means, we make one more observation: An element $\theta \in \Lambda_{i}$ corresponds to a pair of elements of $\Lambda_{i-1}$, and each member of the pair itself corresponds to pairs of elements of $\Lambda_{i-2}$, and so on; so, in the end, an element of $\theta \in \Lambda_{i}$, in fact, corresponds to a sequence of elements of $\Lambda_{1}$ of length $2^{i-1}$. Say the sequence is

$$
\lambda_{1}, \quad \ldots, \quad \lambda_{2}{ }^{i-1} .
$$


Then, the lines it corresponds to all have slope

$$
\lambda_{1} \cdots \lambda_{2^{i-2} / \lambda_{2^{i-2}+1} \cdots \lambda_{2^{i-1}} .}
$$

When our above process terminates at time $t$ satisfying

$$
k<t \ll T,
$$

we will have that the following all hold:

- First, for at least

$$
\left|\Lambda_{1}\right|^{2^{t-2}} n^{-O_{t}(\delta)}
$$

sequences

$$
\lambda_{1}, \ldots, \lambda_{2^{t-2}} \in \Lambda_{1}
$$

we will have a set of lines of slope

$$
\lambda_{1} \cdots \lambda_{2^{t-3}} / \lambda_{2^{t-3}+1} \cdots \lambda_{2^{t-2}}
$$

that are $n^{1-O_{t}(\delta)}$-rich in our grid $A \times A$.

- Second, there are at least

$$
\left|\Lambda_{1}\right|^{2^{t-1}} n^{-O_{t}(\delta)}
$$

pairs of sequences

$$
\lambda_{1}, \ldots, \lambda_{2^{t-1}} \in \Lambda_{1}, \quad \text { and } \quad \lambda_{1}^{\prime}, \ldots, \lambda_{2^{t-1}}^{\prime} \in \Lambda_{1}
$$

corresponding to a pair of elements

$$
\left(v_{1}, v_{2}\right) \in \Lambda_{t-1} \times \Lambda_{t-1},
$$

that are "good for step $t$ " but not "very good for step $t$ " (since otherwise we could continue the iteration for another step). For such a pair, suppose that our $n^{1-O_{t}(\delta)}$-rich lines corresponding to $v_{1}$ are of the form

$$
y=\left(\lambda_{1} \cdots \lambda_{2^{t-3}} / \lambda_{2^{t-3}+1} \cdots \lambda_{2^{t-2}}\right) x+B_{v_{1}},
$$

and those corresponding to $v_{2}$ are of the form

$$
y=\left(\lambda_{1}^{\prime} \cdots \lambda_{2^{t-3}}^{\prime} / \lambda_{2^{t-3}+1}^{\prime} \cdots \lambda_{2^{t-2}}^{\prime}\right) x+B_{\nu_{2}} .
$$

Then, since the pair $\left(v_{1}, v_{2}\right)$ is "good for step $t$ ", we have that there are

$$
\left|B_{v_{1}}\right| \cdot\left|B_{v_{2}}\right| n^{-O_{t}(\delta)}
$$

ordered pairs of lines, one corresponding to $v_{1}$ and the other to $v_{2}$, such that when combined give us an $n^{1-O_{t}(\delta)}$-rich line of the form

$$
y=\alpha x+\left(b_{1}-b_{2}\right) / \beta,
$$


where

$$
\alpha=\lambda_{1} \cdots \lambda_{2^{t-3}} \lambda_{2^{t-3}+1}^{\prime} \cdots \lambda_{2^{t-2}}^{\prime} / \lambda_{1}^{\prime} \cdots \lambda_{2^{t-3}}^{\prime} \lambda_{2^{t-3}+1} \cdots \lambda_{2^{t-2}},
$$

where

$$
b_{1} \in B_{v_{1}}, \quad b_{2} \in B_{v_{2}}, \quad \text { and where } \beta=\lambda_{1}^{\prime} \cdots \lambda_{2^{t-3}}^{\prime} / \lambda_{2^{t-3}+1}^{\prime} \cdots \lambda_{2^{t-2}}^{\prime} .
$$

Furthermore, since the pair $\left(v_{1}, v_{2}\right)$ is not "very good for step $t$ ", we have that the possibilities for the difference $b_{1}-b_{2}$ is at most

$$
n^{\varepsilon\left(1+\delta^{\prime}\right)^{t}} \leq\left|L_{t-1}\left(v_{1}\right)\right|^{1+\delta^{\prime}}=\left|B_{v_{1}}\right|^{1+\delta^{\prime}} .
$$

What this means is that the "additive energy" between the sets $B_{\nu_{1}}$ and $B_{\nu_{2}}$ must be "large". In fact, because there are so many pairs $\left(v_{1}, v_{2}\right)$, there must exist $v_{1} \in \Lambda_{t-1}$ such that there are at least

$$
\left|\Lambda_{t-1}\right| n^{-O_{t}(\delta)}
$$

choices for $v_{2} \in \Lambda_{t}$ such that we have the following lower bound for the additive energy:

$$
\begin{aligned}
E\left(B_{v_{1}}, B_{\nu_{2}}\right) & =\left|\left\{\left(b_{1}, b_{2}, b_{3}, b_{4}\right) \in B_{\nu_{1}} \times B_{\nu_{1}} \times B_{v_{2}} \times B_{\nu_{2}}: b_{1}-b_{3}=b_{2}-b_{4}\right\}\right| \\
& \geq\left|B_{\nu_{1}}\right|^{3-O\left(\delta^{\prime}\right)} .
\end{aligned}
$$

We now require the following standard lemma.

Lemma 2 Suppose that $X$ and $Y$ are sets of size $M$, such that

$$
E(X, Y)=\left|\left\{\left(x, x^{\prime}, y, y^{\prime}\right) \in X \times X \times Y \times Y: x-y=x^{\prime}-y^{\prime}\right\}\right| \geq c M^{3} .
$$

Then, there is some translate $u$ such that

$$
|(X+u) \cap Y| \geq c M .
$$

Proof of the lemma Another way of writing the additive energy is

$$
E(X, Y)=\sum_{\substack{u \in X \\ v \in Y}}|(X-u) \cap(Y-v)| .
$$

So, by simple averaging, among the $M^{2}$ pairs $(u, v) \in X \times Y$, there exists one for which

$$
|(X-u+v) \cap Y|=|(X-u) \cap(Y-v)| \geq c M .
$$

So, for some fixed $v_{1} \in \Lambda_{t-1}$, and for $\left|\Lambda_{t-1}\right| n^{-O_{t}(\delta)}$ elements $v_{2} \in \Lambda_{t-1}$, there exist translates $\tau\left(v_{2}\right)$ for which

$$
\left|B_{v_{1}} \cap\left(B_{\nu_{2}}+\tau\left(v_{2}\right)\right)\right| \geq\left|B_{\nu_{1}}\right| n^{-O_{t}\left(\delta^{\prime}\right)} .
$$

We now arrive at the following basic claim. 
Claim 3 Under the hypotheses of our theorem, there are distinct slopes

$$
\theta_{1}, \quad \ldots, \quad \theta_{N}
$$

where

$$
N>n^{\varepsilon-O(\delta)},
$$

such that for

$$
m=2^{t-2},
$$

at least $N^{m-O(\delta)}$ of the $m$-fold products $\theta_{i_{1}} \cdots \theta_{i_{m}}$, we have a set of $n^{1-O(\delta)}$-rich lines of the form

$$
y=\theta_{i_{1}} \cdots \theta_{i_{m}} x+B\left(i_{1}, \ldots, i_{m}\right),
$$

where $B\left(i_{1}, \ldots, i_{m}\right)$ is some set of slopes. We furthermore assume there is a set $C$ of real numbers such that for each of these $>N^{m-O(\delta)}$ sets $B\left(i_{1}, \ldots, i_{m}\right)$, there exists a real number $\tau\left(i_{1}, \ldots, i_{m}\right)$ such that

$$
\left|B\left(i_{1}, \ldots, i_{m}\right) \triangle\left(C+\tau\left(i_{1}, \ldots, i_{m}\right)\right)\right|<\left|B\left(i_{1}, \ldots, i_{m}\right)\right| n^{-O(\delta)} .
$$

Here, $S \triangle T$ denotes the symmetric difference between $S$ and $T$.

Proof of the claim Basically, we just need to show how these slopes $\theta_{i}$ link up with the lines in (5) and (6); further, we need to explain the presence of the $\delta$ here, rather than the $\delta^{\prime}$ appearing earlier.

Let us first address the issue of the $\delta$ versus of the $\delta^{\prime}$ : Since we get to choose $\delta^{\prime}>0$ as small as desired relative to $\varepsilon>0$, we can just as well rewrite it is $\delta>0$.

As to the relationship between the $\theta_{i}$ 's above and the $\lambda_{j}$ 's in (5), we will take

$$
\left\{\theta_{1}, \ldots, \theta_{N}\right\}=\left\{\lambda_{i}\right\} \cup\left\{1 / \lambda_{i}\right\}
$$

Then, for $m=2^{t-2}$ we have that the lines of (5) have slope of the form $\theta_{i_{1}} \cdots \theta_{i_{m}}$. Furthermore, the fact that $t>k$ is what will allow us to take $m$ as large as needed.

Now we combine together pairs of these rich lines-as discussed in Sect. 2.1having the same slope, to produce many other rich lines having slope 1: Fix one of the slopes $\theta_{i_{1}} \cdots \theta_{i_{m}}$ leading to rich lines with the set of slopes $B\left(i_{1}, \ldots, i_{m}\right)$. Applying Lemma 1 , we find that there are at least

$$
\left|B\left(i_{1}, \ldots, i_{m}\right)\right|^{2} n^{-O(\delta)}
$$

ordered pairs

$$
\left(b, b^{\prime}\right) \in B\left(i_{1}, \ldots, i_{m}\right) \times B\left(i_{1}, \ldots, i_{m}\right)
$$

such that the line

$$
y=x+\left(b-b^{\prime}\right) / \theta_{i_{1}} \cdots \theta_{i_{m}}
$$

is $n^{1-O(\delta)}$-rich in the grid $A \times A$. 
From (7), and a little bit of effort, we can easily deduce that at least $\left|B\left(i_{1}, \ldots, i_{m}\right)\right|^{2}$ $n^{-O(\delta)}$ of these pairs $\left(b, b^{\prime}\right)$ have the property that there exists $\left(c, c^{\prime}\right) \in C \times C$ satisfying

$$
\left(b, b^{\prime}\right)=\left(c+\tau\left(i_{1}, \ldots, i_{m}\right), c^{\prime}+\tau\left(i_{1}, \ldots, i_{m}\right)\right) .
$$

For such pairs, we will have that

$$
b-b^{\prime}=c-c^{\prime} .
$$

By the pigeonhole principle, there exists at least one pair (in fact, lots of pairs) $\left(c, c^{\prime}\right) \in C \times C, c \neq c^{\prime}$, such that at least $N^{m-O(\delta)}$ of the sequences $i_{1}, \ldots, i_{m}$ have the property that the line

$$
y=x+\left(c-c^{\prime}\right) / \theta_{i_{1}} \cdots \theta_{i_{m}}
$$

is $n^{1-O(\delta)}$-rich in the grid $A \times A$. Let us denote this constant $c-c^{\prime}$ as $\xi$, so that our rich lines all look like

$$
y=x+\xi \varphi_{i_{1}} \cdots \varphi_{i_{m}}, \quad \text { where } \varphi_{i}:=1 / \theta_{i} .
$$

By combining together pairs of these lines, as discussed in Sect. 2.1, we can form new ones of the form

$$
y=x+\xi\left(\varphi_{i_{1}} \cdots \varphi_{i_{m}}-\varphi_{j_{1}} \cdots \varphi_{j_{m}}\right)
$$

that are rich in the grid. If we then combine together pairs of those lines, we get ones of the form

$$
y=x+\xi\left(\varphi_{i_{1}} \cdots \varphi_{i_{m}}-\varphi_{j_{1}} \cdots \varphi_{j_{m}}+\varphi_{k_{1}} \cdots \varphi_{k_{m}}-\varphi_{\ell_{1}} \cdots \varphi_{\ell_{m}}\right) .
$$

Continuing in this manner, we can generate lines of slope 1 with $y$-intercept equal to $\xi$ times alternating sums of $m$-fold products of the $\varphi_{i}$ 's; and, at the $t$ th iteration, these alternating sums have $2^{t}$ terms.

\subsection{The Sequence $\Theta_{i}$}

Now we take a digression for a few pages, and define and analyze a certain sequence of expressions: Starting with the set

$$
\Theta:=\left\{\varphi_{i}: i=1,2, \ldots\right\},
$$

consider the sequence of sets (expressions)

$$
\Theta_{1}:=\Theta . \Theta-\Theta . \Theta, \quad \Theta_{2}:=\Theta_{1} . \Theta_{1}-\Theta_{1} . \Theta_{1},
$$

and so on. If we formally expand out the expressions, we will get sums of the following type: $\Theta_{1}$ consists of sums of the type

$$
a_{1} a_{2}-a_{3} a_{4}, \quad a_{i} \in \Theta,
$$


and $\Theta_{2}$ consists of the sums

$$
\begin{aligned}
& a_{1} a_{2} a_{5} a_{6}-a_{3} a_{4} a_{5} a_{6}-a_{1} a_{2} a_{7} a_{8}+a_{3} a_{4} a_{7} a_{8} \\
& \quad-a_{9} a_{10} a_{13} a_{14}+a_{9} a_{10} a_{15} a_{16}+a_{11} a_{12} a_{13} a_{14}-a_{11} a_{12} a_{15} a_{16},
\end{aligned}
$$

where again each $a_{i} \in \Theta$. We will not bother to write down $\Theta_{3}$ ! In general, at the $j$ th iteration, the terms in the alternating sum will involve $4^{j}$ variables $a_{i}$, and the number of terms will be $2^{2^{j}-1}$.

Later on, in another subsection, we will show that so long as $\delta>0$ is small enough, upon expanding $\Theta_{t-2}$ into the alternating sum of products of variables $a_{1}, \ldots, a_{4^{t-2}}$, as in (10) and (11), at least

$$
|\Theta|^{4^{t-2}} n^{-O_{t}(\delta)}
$$

choices for these $a_{i} \in \Theta$ will produce a

$$
\theta=\theta\left(a_{1}, \ldots, a_{4^{t-2}}\right) \in \Theta_{t-2}
$$

so that the line

$$
y=x+\xi \theta
$$

is $n^{1-O_{t}(\delta)}$-rich in the grid $A \times A$. We will then use Lemma 3 to show that this is impossible for $t$ large enough and $\delta>0$ small enough. The fact that $t>k$, where $k$ is chosen as large as desired ( $k$ is as appears in Sect. 2.2), will allow us to reach our contradiction, thereby proving Theorem 2 .

\subsubsection{A Certain Inductive Claim}

The key fact that we will show and use to accomplish our goal is the following.

Claim 4 Suppose that $g\left(x_{1}, \ldots, x_{u}\right)$ is some polynomial in the variables $x_{1}, \ldots, x_{u}$ which are to be thought of as taking on values in the set $\Theta$. Consider the expansion of

$$
\Theta_{j} \Theta_{j} g\left(x_{1}, \ldots, x_{u}\right)
$$

into the variables $a_{1}, \ldots, a_{2 \cdot 4^{j}}, x_{1}, \ldots, x_{u} \in \Theta .{ }^{1}$ Suppose that there are at least

$$
|\Theta|^{2 \cdot 4^{j}+u} n^{-O_{j, u}(\delta)}
$$

choices for these variables, producing a value

$$
\gamma=\gamma\left(a_{1}, \ldots, x_{u}\right)=\Theta_{j} \Theta_{j} g\left(x_{1}, \ldots, x_{u}\right)
$$

such that the line

$$
y=x+\xi \gamma
$$

\footnotetext{
${ }^{1}$ The first $\Theta_{j}$ is expanded into $a_{1}, \ldots, a_{4 j}$, and the second $\Theta_{j}$ is expanded into $a_{4 j+1}, \ldots, a_{2 \cdot 4^{j}}$.
} 
is $n^{1-O_{j, u}(\delta)}$-rich in the grid $A \times A$. Then, there are at least

$$
|\Theta|^{4^{j+1}+u} n^{-O_{j, u}(\delta)}
$$

choices for the variables

$$
b_{1}, \ldots, b_{4^{j+1}}, y_{1}, \ldots, y_{u} \in \Theta
$$

such that the line

$$
y=x+\xi \gamma^{\prime}, \quad \gamma^{\prime}=\gamma^{\prime}\left(b_{1}, \ldots, y_{u}\right) \in \Theta_{j+1} g\left(y_{1}, \ldots, y_{u}\right)
$$

is $n^{1-O_{j, u}(\delta)}$-rich in $A \times A$.

Proof of the claim Under the hypotheses of the above claim, the pigeonhole principle implies that for at least

$$
|\Theta|^{4^{j+1}+u} n^{-O_{j, u}(\delta)}
$$

choices of variables

$$
b_{1}, \ldots, b_{2 \cdot 4^{j}}, c_{1}, \ldots, c_{2 \cdot 4^{j}}, x_{1}, \ldots, x_{u} \in \Theta
$$

we will have that if we let

$$
\gamma_{1}:=\gamma_{1}\left(b_{1}, \ldots, b_{2 \cdot 4^{j}}, x_{1}, \ldots, x_{u}\right) \in \Theta_{j} \Theta_{j} g\left(x_{1}, \ldots, x_{u}\right),
$$

and

$$
\gamma_{2}:=\gamma_{2}\left(c_{1}, \ldots, c_{2 \cdot 4^{j}}, x_{1}, \ldots, x_{u}\right) \in \Theta_{j} \Theta_{j} g\left(x_{1}, \ldots, x_{u}\right)
$$

(note that the value of $x_{1}, \ldots, x_{u}$ here is the same as for $\gamma_{1}$ ), then both the lines

$$
y=x+\xi \gamma_{1} \quad \text { and } \quad y=x+\xi \gamma_{2}
$$

are $n^{1-O_{j, u}(\delta)}$-rich in $A \times A$. Furthermore, by dint of Lemma 1 and the comments following it, we will additionally have that for (13) many choices of the $b_{i}$ 's, $c_{i}$ 's, and $x_{i}$ 's, the pair of lines may be combined to produce the new line

$$
y=x+\xi\left(\gamma_{1}-\gamma_{2}\right),
$$

which will also be $n^{1-O_{j, u}(\delta)}$-rich in $A \times A$.

This

$$
\gamma_{1}-\gamma_{2}=\left(\Theta_{j} \Theta_{j}-\Theta_{j} \Theta_{j}\right) g\left(x_{1}, \ldots, x_{u}\right)
$$

has the form $\Theta_{j+1} g\left(x_{1}, \ldots, x_{u}\right)$. Clearly, this proves the claim.

A consequence of this claim, and an easy induction argument (to be described presently), is that if the number of choices for

$$
x_{1}, \ldots, x_{2} z \in \Theta
$$


for which

$$
y=x+\xi x_{1} \cdots x_{2} z
$$

is $n^{1-O_{Z}(\delta)}$-rich in $A \times A$ is at least

$$
|\Theta|^{2^{Z}} n^{-O_{Z}(\delta)}
$$

which it is by the properties of the set $\Theta$ described earlier, then there are at least

$$
|\Theta|^{4^{Z}} n^{-O_{Z}(\delta)}
$$

choices for $y_{1}, \ldots, y_{4} z \in \Theta$ such that the line

$$
y=x+\xi \gamma, \quad \gamma=\gamma\left(y_{1}, \ldots, y_{4} z\right) \in \Theta_{Z}
$$

is $n^{1-O_{Z}(\delta)}$-rich in $A \times A$.

The way that this is proved is as follows: First, write the product

$$
x_{1} \cdots x_{2} z=\left(x_{1} x_{2}\right)\left(x_{3} x_{4}\right) \cdots\left(x_{2} z_{-1} x_{2} z\right) .
$$

Then, applying the claim to the pair $x_{1} x_{2}$, and then $x_{3} x_{4}$, and so on, we deduce that lots of variable choices make lines $y=x+\xi \alpha, \alpha \in \Theta_{1} \cdots \Theta_{1}\left(2^{Z-1}\right.$ copies here), rich in $A \times A$. Then, the claim is applied again to the products $\Theta_{1} \Theta_{1}$ (grouped in twos), leading to lines $y=x+\xi \beta, \beta \in \Theta_{2} \cdots \Theta_{2}\left(2^{Z-2}\right.$ copies here). Continuing, one reaches lines $y=x+\xi \gamma, \gamma \in \Theta_{Z}$, as claimed.

Combining this deduction with Claim 3, we deduce:

Claim 5 There are at least

$$
N^{4^{t-2}-O_{t}(\delta)}
$$

choices of variables $a_{1}, \ldots, a_{4^{t-2}} \in \Theta$ such that for $\theta=\theta\left(a_{1}, \ldots, a_{4^{t-2}}\right) \in \Theta_{t-2}$, the line

$$
y=x+\xi \theta
$$

is $n^{1-O_{t}(\delta)}$-rich in $A \times A$.

\subsection{A Growth Lemma}

Given a probability measure $f$ supported on a finite set $C$, we let $f^{*}$ denote a certain measure on $C C-C C$ given as follows:

$$
f^{*}(x):=\sum_{c_{1} c_{2}-c_{3} c_{4}=x} f\left(c_{1}\right) f\left(c_{2}\right) f\left(c_{3}\right) f\left(c_{4}\right) .
$$

Lemma 3 Suppose that $C$ is a finite set of real numbers. Let $f$ be a measure on $C$. Then,

$$
\max _{x} f^{*}(x) \ll\left(\max _{x} f(x)\right)^{4 / 3}(\log |C|)^{2}
$$




\subsubsection{Proof of Lemma 3}

Let

$$
M:=\max _{x} f(x) .
$$

We begin by partitioning the set $C$ into the disjoint sets, some of which may be empty:

$$
C=C_{1} \cup C_{2} \cup \cdots \cup C_{k} \cup C_{0},
$$

where for $i \geq 1$,

$$
C_{i}:=\left\{c \in C: f(c) \in\left(2^{-i} M, 2^{-i+1} M\right]\right\},
$$

where $C_{0}$ is the remaining elements of $C$, and where

$$
k=\lfloor 5 \log |C| / \log 2\rfloor+1 .
$$

We define

$$
f_{\alpha, \beta, \gamma, \delta}^{*}(x):=\sum_{\substack{c_{1} \in C_{\alpha}, c_{2} \in C_{\beta}, c_{3} \in C_{\gamma}, c_{4} \in C_{\delta} \\ c_{1} c_{2}-c_{3} c_{4}=x}} f\left(c_{1}\right) f\left(c_{2}\right) f\left(c_{3}\right) f\left(c_{4}\right) .
$$

We have that

$$
f^{*}(x)=\sum_{0 \leq \alpha, \beta, \gamma, \delta \leq k} f_{\alpha, \beta, \gamma, \delta}^{*}(x) .
$$

To prove the theorem, then, all we need to do is get bounds on these individual terms, and then sum them up.

First, we can easily bound the total contribution of the terms where any of the $\alpha, \beta, \gamma$, or $\delta$ is 0 : The contribution of all such terms is clearly bounded from above by

$$
\ll \sum_{x \in C C-C C} M 2^{-5 \log |C| / \log 2} \ll|C|^{-1} .
$$

Now we handle the other terms. First, suppose that $1 \leq \alpha, \beta, \gamma, \delta \leq k$. Then, one easily sees from the fact $f$ is a probability measure that

$$
\left|C_{i}\right| \ll 2^{i} M^{-1}, \quad i=\alpha, \beta, \gamma, \delta .
$$

The size of $f_{\alpha, \beta, \gamma, \delta}^{*}(x)$ is

$$
\ll M^{4} 2^{-\alpha-\beta-\gamma-\delta}\left|\left\{a \in C_{\alpha}, b \in C_{\beta}, c \in C_{\gamma}, d \in C_{\delta}: a b-c d=x\right\}\right| .
$$

To bound this last factor from above, we will apply Elekes's [3] idea of using the Szemerédi-Trotter incidence theorem [7] to prove sum-product inequalities. We begin with the Szemerédi-Trotter theorem: 
Theorem 3 Suppose that one has $N$ points and L lines in the plane. Then, the number of incidences is bounded from above by

$$
O\left((N L)^{2 / 3}+N+L\right) .
$$

The way we apply this theorem is as follows: Consider the family of lines

$$
a x+c y=z, \quad \text { where } a \in C_{\alpha}, c \in C_{\gamma} .
$$

Note that there are $\left|C_{\alpha}\right| \cdot\left|C_{\gamma}\right|$ lines in total.

Each of these lines intersects the grid $C_{\beta} \times C_{\delta}$ in some number of points (or perhaps no points at all). The total number of incidences $(x, y) \in C_{\beta} \times C_{\delta}$ is the right-most factor of (17). From the Szemerédi-Trotter theorem, this number is

$$
\begin{aligned}
& \ll\left(\left|C_{\alpha}\right| \cdot\left|C_{\beta}\right| \cdot\left|C_{\gamma}\right| \cdot\left|C_{\delta}\right|\right)^{2 / 3}+\left|C_{\beta}\right| \cdot\left|C_{\delta}\right|+\left|C_{\alpha}\right| \cdot\left|C_{\gamma}\right| \\
& \ll 2^{2(\alpha+\beta+\gamma+\delta) / 3} M^{-8 / 3}+2^{\beta+\delta} M^{-2}+2^{\alpha+\gamma} M^{-2} .
\end{aligned}
$$

The total weight $f(a) f(x) f(c) f(y)$ that each such representation $a x+c y=z$ gets is

$$
\ll 2^{-\alpha-\beta-\gamma-\delta} M^{4}
$$

So,

$$
f_{\alpha, \beta, \gamma, \delta}^{*}(z) \ll 2^{-(\alpha+\beta+\gamma+\delta) / 3} M^{4 / 3}+2^{-\alpha-\gamma} M^{2}+2^{-\beta-\delta} M^{2} .
$$

It follows that for all $z \in C C-C C$,

$$
f^{*}(z) \ll|C|^{-1}+M^{4 / 3}(\log |C|)^{2} \ll M^{4 / 3}(\log |C|)^{2} .
$$

The second inequality here comes from the fact that $M \geq|C|^{-1}$, which follows from the fact that $f$ is a probability measure.

\subsection{Continuation of the Proof}

We now define a sequence of functions by first letting

$$
f_{0}(h):= \begin{cases}1 / N, & \text { if } h \in \Theta ; \\ 0, & \text { if } h \notin \Theta .\end{cases}
$$

(Note that $f_{0}$ is a probability measure.) Then, we inductively define

$$
f_{i+1}(h):=f_{i}^{*}(h),
$$

where $f^{*}$ is as in (16). It is easy to see that these $f_{i}$ are all also probability measures.

The connection between this function $f$ and our sequence of $\Theta_{i}$ is as follows: For a given real number $h$, we have that $f_{j}(h)$ is $|\Theta|^{-4^{j}}$ times the number of choices for

$$
x_{1}, \ldots, x_{4^{j}} \in \Theta
$$


such that

$$
\theta=\theta\left(x_{1}, \ldots, x_{4^{j}}\right) \in \Theta_{j}
$$

satisfies

$$
\theta=h \text {. }
$$

As we will see, the upper bound on $f_{j}(h)$ provided by Lemma 3 will produce for us a lower bound on the number of rich lines in our grid.

Now, Lemma 3 implies that for some constant $c>0$, if

$$
t \geq k:=c \log (1 / \varepsilon)
$$

then for all $h$,

$$
f_{t-2}^{*}(h) \leq 1 / n^{5}
$$

So, for each real number $h$, there are at most

$$
n^{-5}|\Theta|^{4^{t-2}}
$$

choices for $x_{1}, \ldots, x_{4^{t-2}} \in \Theta$ such that $\theta=\theta\left(x_{1}, \ldots, x_{4^{t-2}}\right)$ equals $h$. Combining this with Claim 5, we quickly deduce that there are $n^{5-O_{t}(\delta)}$ distinct values of $\theta$ among these rich lines (of Claim 5). If $\delta>0$ is small enough relative to $\varepsilon$, then we will see that this number exceeds $n^{4}$.

We have now reached a contradiction, since there can be at most $n^{4}$ lines that hit an $n \times n$ grid in at least two points each. Our theorem is now proved.

Acknowledgements We would like to thank Boris Bukh, Jozsef Solymosi, B. Sudakov, P.M. Wood, T. Tao, H. Helfgott, and A. Granville.

\section{References}

1. Bourgain, J., Katz, N., Tao, T.: A sum-product estimate in finite fields, and applications. Geom. Funct. Anal. 14, 27-57 (2004)

2. Erdős, P., Szemerédi, E.: On sums and products of integers. In: Studies in Pure Mathematics, pp. 213218. Birkhäuser, Basel (1983)

3. Elekes, G.: On the number of sums and products. Acta Arith. 81, 365-367 (1997)

4. Elekes, G.: Sums versus products in number theory, algebra and Erdős geometry. Paul Erdős and His Mathematics, II (Budapest, 1999), Bolyai Soc. Math. Stud., vol. 11, pp. 241-290, János Bolyai Math. Soc. (2002)

5. Helfgott, H.: Growth and generation in $S L_{2}(\mathbb{Z} / p \mathbb{Z})$. Ann. Math. 167, 601-623 (2008)

6. Helfgott, H.: Growth and generation in $S L_{3}(\mathbb{Z} / p \mathbb{Z})$. To appear in J. Eur. Math. Soc.

7. Szemerédi, E., Trotter, W.T.: Extremal problems in discrete geometry. Combinatorica 3, 381-392 (1983)

8. Wood, M.M., Wood, P.M., Vu, V.: Mapping incidences. arXiv:0711.4407 